\section{Musculoskeletal Static Workspace Analysis of the Human Shoulder as a Cable-Driven Robot}

Darwin Lau, Member, IEEE, Jonathan Eden Student Member, IEEE, Denny Oetomo, Senior Member, IEEE, and Saman K. Halgamuge, Senior Member, IEEE

\begin{abstract}
The workspace analysis of cable-driven parallel manipulators has been widely studied, where the cables have been considered as ideal force generators. Due to the differences in actuation dynamics, workspace analysis has not been previously conducted for musculoskeletal systems. In this paper, static workspace analysis is performed on the human shoulder with a Hill-type physiological muscle model. The key characteristic of physiological muscles is that its ability to produce force is dependent on its length. Such type of workspace analysis on musculoskeletal systems as cable-driven manipulators is proposed for the first time. The generated shoulder workspace is validated by comparing the range of motion to that from benchmarks of human data. The significance of considering physiological muscles is demonstrated by comparing the musculoskeletal workspace with that of systems with ideal force generators. The novel formulation provides a new computational approach to perform workspace analysis for a wider range of engineered and biological systems.
\end{abstract}

\section{INTRODUCTION}

Cable-driven parallel manipulators are articulated mechanisms actuated by cables arranged in parallel configuration. The key characteristic of cable-driven systems is that cables can only provide actuation unilaterally in tension (positive cable force). Cable-driven manipulators can be regarded as bio-inspired systems [1], [2] , where the muscles and bones are anthropomorphically analogous to the cables and rigid links, respectively. Furthermore, both cables and muscles can only provide unilateral actuation. However, one major difference between the two types of systems is the actuation dynamics in the cables and muscles.

In previous studies of cable-driven manipulators, cables have been modelled as ideal force generators, which assumes that any magnitude of positive cable force within a given range can be produced. The range of producible cable force is considered as a property of the actuator with lower and upper bounds that are constant for all manipulator poses. The ideal force generator model fails when dealing with systems where the ability to produce force is dependent on the position and velocity of the system (state dependent force generator). State dependent force generators exist in many engineered and biological systems, such as those actuated by pneumatic artificial muscles [3] and physiological muscles [4], respectively. For example, the force that can be produced by a physiological muscle varies depending on its length [4].

In robotics, workspace analysis is a technique that allows the manipulator's operational region to be computationally generated. Workspace analysis has been well studied for

D. Lau, D. Oetomo, S. K. Halgamuge are with the Department of Mechanical Engineering, The University of Melbourne, Victoria, Australia laudtaunimelb.edu.au, j. eden2 a student . unimelb. edu . au, $\{$ doetomo, saman\} aunimelb. edu. au traditional serial rigid link manipulators [5]-[7]. For cabledriven manipulators, many types of workspace exist due to the positive cable force constraint, such as static workspace [8], wrench-closure workspace [9] and wrench-feasible workspace [10]. Static workspace refers to the set of poses in which the manipulator can achieve static equilibrium, defined as the ability of the manipulator to sustain its own gravity force under no external wrenches.

Robotics workspace analysis techniques cannot be directly applied onto musculoskeletal systems as previous studies have assumed ideal force generators, resulting in inaccurately generated workspace. In biomechanical studies, human joint limits and muscle function have been experimentally investigated in vivo, where vision systems [11] or motion sensors [12], [13] are used to record the motion of human subjects. There are two major limitations with experimental approaches. Firstly, the recorded motion only represents a subset of the true operational region, since poses that are not observed do not imply that it is unreachable by the subject. Secondly, this approach does not allow the effects of changes in muscle properties and their attachment locations on the operational region to be predicted.

In this paper, the static workspace analysis of a human shoulder is presented. The analysis is performed by considering the musculoskeletal system as a cable-driven parallel manipulator that possesses cables with the actuation characteristics of physiological muscles. The model assumes that the cables' ability to actuate is dependent on the system pose, and also can behave as active or passive elements. The resulting workspace is validated against the range of motion data reported from human benchmarks from the literature. Furthermore, the workspace is compared with that of a cabledriven system with ideal force generators. The results show that the proposed technique is capable of computationally generating a workspace that is physiologically accurate.

The primary motivation of this study is to increase the understanding of human biomechanics through the static workspace analysis of musculoskeletal systems. Workspace analysis for such systems addresses the issues involved with existing techniques in studying the limits of human joints and can be beneficial for a range of applications in rehabilitation robotics and biomechatronics. For example, in the treatment of upper limb post-stroke rehabilitation, the understanding of muscle contributions to the reachable workspace of the shoulder would eventually allow more targeted rehabilitation treatment of motion impairment. Furthermore, in tendontransfer surgery, the effects of muscle relocation can be computationally simulated. This surgery is performed to help recover the lost range of motion in a subject, for example due to a shoulder rotator-cuff tear.

The remainder of the paper is organised as follows: Section II presents the workspace analysis framework. The musculoskeletal static workspace with a physiological muscle model is introduced in Section III. The human shoulder model used in the workspace analysis is described in Section IV. The results of the shoulder workspace is presented in Sections $\mathrm{V}$ and the results are discussed in Section VI. Finally, Section VII concludes the paper and presents areas of future work. 


\section{Static Workspace Analysis}

The equations of motion of an $n$ degrees-of-freedom cabledriven manipulator actuated by $m$ cables can be expressed as

$$
M(\mathbf{q}) \ddot{\mathbf{q}}+\mathbf{C}(\mathbf{q}, \dot{\mathbf{q}})+\mathbf{G}(\mathbf{q})+\mathbf{F}_{e x t}=-J^{T}(\mathbf{q}) \mathbf{f},
$$

where $M, \mathbf{C}, \mathbf{G}$, and $\mathbf{F}_{e x t}$ correspond to the mass inertia matrix, centrifugal and Coriolis force vector, gravitational vector, and external wrenches, respectively. The vector of cable forces is denoted by $\mathbf{f}=\left[\begin{array}{llll}f_{1} & f_{2} & \ldots & f_{m}\end{array}\right]^{T} \in \mathbb{R}^{m}$ where $f_{i} \geq 0$ represents the cable force in cable $i$. The manipulator pose is represented by $\mathbf{q}=\left[\begin{array}{lll}q_{1} & \ldots & q_{n}\end{array}\right]^{T} \in \mathbb{R}^{n}$. The resultant wrench due to cable forces is denoted by $-J^{T} \mathbf{f}$, where $J^{T}(\mathbf{q}) \in \mathbb{R}^{n \times m}$ is the transpose of the Jacobian matrix.

\section{A. Ideal Force Generator Model}

The static workspace $(S W)$ for cable-driven systems is defined as the set of poses in which the manipulator can sustain its own weight given the actuation forces, under no motion $(\dot{\mathbf{q}}=\ddot{\mathbf{q}}=\mathbf{0})$ and no external wrenches $\left(\mathbf{F}_{e x t}=\mathbf{0}\right)$. Hence, from the equations of motion in (1), the static workspace for cable-driven systems can be defined as

$$
S W=\left\{\mathbf{q}: \mathbf{G}(\mathbf{q})=-J^{T}(\mathbf{q}) \mathbf{f}, \exists \mathbf{f} \in\left[\mathbf{0}, \mathbf{f}_{\max }\right]\right\},
$$

where $\mathbf{f}_{\text {max }}=\left[\begin{array}{llll}f_{1, \max } & f_{2, \max } & \ldots & f_{\text {m, max }}\end{array}\right]^{T} \in \mathbb{R}^{m}$ represents the maximum allowable force for each cable. The workspace definition from (2) assumes that the cables are ideal force generators, meaning that the cables have the ability to produce any force up to fixed maximum values for any manipulator pose.

Typically, the static workspace from (2) can be generated through analytical or numerical approaches. In numerical approaches, a discrete search space $\mathcal{Q}$ is defined and a pose $\mathbf{q} \in \mathcal{Q}$ can be considered as being within the static workspace if the optimisation problem

$$
\begin{aligned}
\mathbf{f}^{*}= & \underset{\mathbf{f}}{\arg \min } c(\mathbf{f}) \\
\text { s.t. } & \mathbf{G}(\mathbf{q})=-J^{T}(\mathbf{q}) \mathbf{f} \\
& \mathbf{0} \leq \mathbf{f} \leq \mathbf{f}_{\max }
\end{aligned}
$$

yields a valid solution for any cost function $c(\mathbf{f})$. Since workspace analysis is only concerned with the existence of solutions to the workspace condition, any cost function $c(\mathbf{f})$ can be used. Hence, the existence of solutions to (3) can be efficiently solved by selecting the linear cost function $c(\mathbf{f})=\mathbf{f}$, resulting in a linear programming problem.

\section{B. State Dependent Force Generator Model}

The assumption of cables as ideal force generators may not be valid for actuators that produce force depending on the system states. To perform workspace analysis on this class of systems, a more generalised cable model is introduced with two key features. Firstly, cables can be considered as active or passive elements depending on its length. When a cable is active, it can be actuated within a range of positive cable force, such that $0 \leq f_{i, \min } \leq f_{i} \leq f_{i, \max }$. Otherwise, the cable is passive and constrained to have a fixed actuation force $f_{i}=f_{p} \geq 0$. Note that the definition of passive cable in this context does not imply that no force is produced on the cable. Secondly, the active cable force range and passive cable force are also dependent on its cable length at pose q.

For a system with $m$ cables $m=m_{a}+m_{p}, m_{a}$ and $m_{p}$ represent the number of active and passive cables, respectively. The set of cable forces is comprised of active and passive cable forces $\mathbf{f}=\left\{\mathbf{f}_{a}, \mathbf{f}_{p}\right\}$, where $\mathbf{f}_{a} \in \mathbb{R}^{m_{a}}$ and $\mathbf{f}_{p} \in \mathbb{R}^{m_{p}}$ represent the set of active cable forces and the set of passive cable forces, respectively. The columns of $J^{T}$ can be rearranged such that

$$
J^{T} \mathbf{f}=\left[\begin{array}{ll}
J_{a}^{T} & J_{p}^{T}
\end{array}\right]\left[\begin{array}{l}
\mathbf{f}_{a} \\
\mathbf{f}_{p}
\end{array}\right],
$$

where $J_{a}^{T} \in \mathbb{R}^{n \times m_{a}}$ and $J_{p}^{T} \in \mathbb{R}^{n \times m_{p}}$ are formed from the columns of $J^{T}$ for the active and passive cables, respectively.

The minimum and maximum allowable cable force for active cables can be denoted by vectors $\underline{\mathbf{f}}_{a} \in \mathbb{R}^{m_{a}}$ and $\overline{\mathbf{f}_{a}} \in \mathbb{R}^{m_{a}}$, respectively, and hence $\mathbf{f}_{a} \in\left[\underline{\overline{\mathbf{f}_{a}}}(\mathbf{q}), \overline{\mathbf{f}_{a}}(\mathbf{q})\right]$. It should be noted that for cable-driven systems, the length $l_{i}$ of cable $i$ is dependent on the pose of the system q. Substitution of (4) into (2) results in a static workspace definition where cables are state dependent force actuators

$$
S W^{*}=\left\{\mathbf{q}: \mathbf{G}(\mathbf{q})+J_{p}^{T} \mathbf{f}_{p}=-J_{a}^{T} \mathbf{f}_{a}, \exists \mathbf{f}_{a} \in\left[\underline{\mathbf{f}_{a}}, \overline{\mathbf{f}_{a}}\right]\right\} .
$$

Several key differences can be observed between the definition of the static workspace with ideal force generators in (2) and state dependent force generators in (5). Firstly, the set of active cables is always a subset of the cables for the system, resulting in a reduced number of actuators for a system with state dependent force generators compared to that with ideal force generators. Secondly, the force range $\mathbf{f} \in\left[\mathbf{0}, \mathbf{f}_{\max }\right]$ from (2) implies that cables can produce any force within the constant force range for all manipulator poses. In comparison, $\mathbf{f}_{a} \in\left[\underline{\mathbf{f}}_{a}(\mathbf{q}), \overline{\mathbf{f}_{a}}(\mathbf{q})\right]$ allows for a varying range of actuation that is dependent on the manipulator pose. Thirdly, the passive cable forces produce a resultant wrench $J_{p}^{T} \mathbf{f}_{p}$ that must also be counteracted by the active forces to achieve static equilibrium.

Similar to (3), the pose $q$ can be considered as being within the workspace $S W^{*}$ defined in (5) if a solution exists to

$$
\begin{aligned}
\mathbf{f}_{a}^{*}= & \underset{\mathbf{f}_{a}}{\arg \min } \mathbf{f}_{a} \\
\text { s.t. } & \mathbf{G}(\mathbf{q})+J_{p}^{T} \mathbf{f}_{p}=-J_{a}^{T} \mathbf{f}_{a} \\
& \underline{\mathbf{f}_{a}} \leq \mathbf{f}_{a} \leq \overline{\mathbf{f}_{a} .} .
\end{aligned}
$$

\section{Musculoskeletal Static Workspace}

\section{A. Muscle Model}

One widely accepted model of the physiological muscle is the modified Hill-type model [4] consisting of tendon and muscle elements connected in series, as shown in Figure 1. The combined muscle-tendon length $l^{m t}$ can be expressed with respect to the tendon length $l^{t}$, muscle length $l^{m}$ and muscle pennation angle $\alpha$ by

$$
l^{m t}=l^{t}+l^{m} \cos \alpha .
$$

The force applied along the muscle-tendon complex is transmitted through both the muscle and tendon elements in 


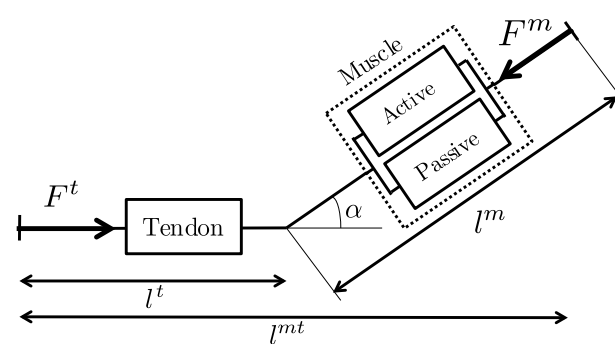

Fig. 1. Model of the Hill-type muscle-tendon complex

series. Hence, the muscle-tendon force $F^{m t}$ can be related to the tendon force $F^{t}$ and the muscle force $F^{m}$ by

$$
F^{m t}=F^{t}=F^{m} \cos \alpha .
$$

The relationship between the force that can be produced and the muscle-tendon length can be described by a set of generic force relationships and muscle specific properties [14]: peak isometric muscle force $F_{0}^{m}$, optimal muscle fibre length $l_{0}^{m}$, optimal muscle fibre pennation angle $\alpha_{0}$ and tendon slack length $l_{s}^{t}$.

The tendon behaves as a passive non-linear elastic element. One model for the generic tendon force-strain relationship [14] can be analytically expressed as

$$
\hat{F}^{t}(\varepsilon)=\left\{\begin{array}{cc}
0 & \varepsilon<0 \\
0.10377\left(e^{91 \varepsilon}-1\right) & 0 \leq \varepsilon<0.01516 \\
37.526 \varepsilon-0.26029 & 0.01516 \leq \varepsilon<0.1
\end{array},\right.
$$

where $\hat{F}^{t}$ and $\varepsilon$ are the normalised tendon force and tendon strain, respectively. Tendon strain is defined by $\varepsilon=\left(l^{t}-l_{s}^{t}\right) / l_{s}^{t}$ and normalised tendon force is $\hat{F}^{t}=F^{t} / F_{0}^{m}$.

As shown in Figure 1, the muscle element consists of two components: an active component capable of producing force $\hat{F}_{a}^{m}$ and a passive component that produces force $\hat{F}_{p}^{m}$. Assuming that the system is in static equilibrium, and hence $i^{m}=0$ for all muscles, the normalised muscle force $\hat{F}^{m}=F^{m} / F_{0}^{m}$ can be expressed as

$$
\hat{F}^{m}(\eta)=\hat{F}_{a}^{m}(\eta) a(t)+\hat{F}_{p}^{m}(\eta),
$$

where $\eta=l^{m} / l_{0}^{m}$ is the normalised muscle length and $0 \leq$ $a(t) \leq 1$ is the activation level of the muscle at time $t$.

The active muscle force $\hat{F}_{a}^{m}(\eta)$ [14] and passive muscle force $\hat{F}_{p}^{m}(\eta)$ [15] relationships can be expressed as

$$
\begin{aligned}
& \hat{F}_{a}^{m}(\eta)=\left\{\begin{array}{cc}
1-\left(\frac{\eta-1}{0.5}\right)^{2} & 0.5<\eta<1.5 \\
0 & \text { otherwise }
\end{array}\right. \\
& \hat{F}_{p}^{m}(\eta)=\eta^{3} e^{8 \eta-12.9} .
\end{aligned}
$$

The muscle is considered as an active muscle for $0.5<\eta<$ 1.5 , where $\hat{F}_{a}^{m}>0$, such that the muscle can be activated. Otherwise, the muscle is considered as a passive muscle where $\hat{F}_{a}^{m}=0$ and hence $\hat{F}^{m}=\hat{F}_{p}^{m}$. Assuming that the muscle belly has a constant thickness and volume [16], the pennation angle $\alpha(t)$ can be expressed as

$$
\alpha(t)=\sin ^{-1}\left(\frac{l_{0}^{m}}{l^{m}} \sin \alpha_{0}\right) .
$$

\section{B. Determination of Muscle Force Range}

From kinematics analysis, the muscle origin and insertion points can only provide the length of the entire muscle-tendon complex $l^{m t}$. For static equilibrium $\dot{a}(t)=0$, the lengths of the muscle and tendon elements can be resolved from the muscletendon length $l^{m t}$. The resulting muscle length $l^{m}$ allows the muscle-tendon force $F^{m t}$ to be determined.

Substituting (13) into (8) and (7) results in the relationships

$$
\begin{aligned}
\hat{F}^{t}(\varepsilon) & =\left(\hat{F}_{a}^{m}(\eta) a(t)+\hat{F}_{p}^{m}(\eta)\right) \sqrt{1-\frac{\sin ^{2} \alpha_{0}}{\eta^{2}}}, \\
\varepsilon & =\frac{l^{m t}-l_{0}^{m} \sqrt{\eta^{2}-\sin ^{2} \alpha_{0}}-l_{s}^{t}}{l_{s}^{t}} .
\end{aligned}
$$

For a particular $l^{m t}$, the solution to (14) and (15) allows the admissible range of muscle-tendon forces $F^{m t}$ to be determined. Figure 2 shows the curves for $F^{m} \cos \alpha$ and $F^{t}(\eta)$ for an example muscle, where curves $F_{1}^{t}$ to $F_{5}^{t}$ represent $F^{t}(\eta)$ for increasing values of $l^{m t}$. For the muscle-force curves $F^{m} \cos \alpha$, several activation levels between $a=0$ and $a=1$ are shown.

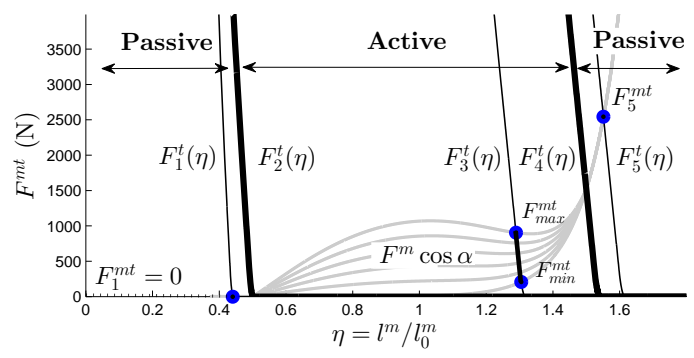

Fig. 2. Muscle-tendon forces for a range of $l^{m t}$, showing the scenarios in which the muscle is active and passive

If the solution for $\eta$ to (14) is within $0.5<\eta<1.5$, for example $F_{3}^{t}(\eta)$ in Figure 2, then the muscle is considered active and a range of forces can be produced as the activation varies between $a=0$ and $a=1$. In contrast, if the solution for $\eta$ to (14) lies within $\eta \leq 0.5$ or $\eta \geq 1.5$, as shown by example curves $F_{1}^{t}(\eta)$ and $F_{5}^{t}(\eta)$, respectively, there is only one $F^{m t}$ solution and the muscle is passive.

It can be observed that the increase in $l^{m t}$ translates the curve $F^{t}(\eta)$ monotonically from left to right. The muscletendon lengths $l_{a, \min }^{m t}$ for $F_{2}^{t}(\eta)$ and $l_{a, \max }^{m t}$ for $F_{4}^{t}(\eta)$ can be regarded as the minimum and maximum muscle-tendon lengths, respectively. The minimum active muscle-tendon length can be defined as the muscle-tendon length in which $\varepsilon=0$ and $\eta=0.5$. Hence from (15), $l_{a, \min }^{m t}$ can be expressed as

$$
l_{a, \min }^{m t}=l_{0}^{m} \sqrt{(0.5)^{2}-\sin ^{2} \alpha_{0}}+l_{s}^{t} .
$$

The maximum active muscle-tendon length can be defined as the value of $l^{m t}$ such that (14) is satisfied for $\eta=1.5$

$$
\hat{F}^{t}(\eta=1.5)=\hat{F}_{p}^{m}(\eta=1.5) \sqrt{1-\frac{\sin ^{2} \alpha_{0}}{1.5^{2}}}
$$

The solution to (17) can be determined using the tendon force 
and passive muscle force relationships from (9) and (12), respectively. The normalised tendon force at $\eta=1.5$ is

$$
\hat{F}_{\text {max }}^{t}=1.5^{2} e^{-0.9} \sqrt{1.5^{2}-\sin ^{2} \alpha_{0}} .
$$

Equating (15) and (18) allows the maximum active muscletendon length to be determined as

$$
l_{a, \max }^{m t}=\frac{\left(\hat{F}_{\max }^{t}+37.7863\right) l_{s}^{t}}{37.526}+l_{0}^{m} \sqrt{1.5^{2}-\sin ^{2} \alpha_{0}} .
$$

When a muscle is active, the muscle-tendon complex can produce a force within the range of $F^{m t} \in\left[F_{\text {min }}^{m t}, F_{\text {max }}^{m t}\right]$. From the curves $F_{3}^{t}(\eta)$ and $F^{m} \cos \alpha$ shown in Figure 2, it can be observed that $F_{m i n}^{m t}$ and $F_{m a x}^{m t}$ can be determined by solving (14) for $a(t)=0$ and $a(t)=1$, respectively. As a result, the active muscle force range can be expressed as

$$
\begin{aligned}
& F_{\text {min }}^{\text {mit }}=F_{0}^{m} \hat{F}^{t}\left(\eta=\eta_{\text {min }}^{*}\right) \\
& F_{\text {max }}^{m t}=F_{0}^{m} \hat{F}^{t}\left(\eta=\eta_{\max }^{*}\right),
\end{aligned}
$$

where $\eta_{\min }^{*}$ and $\eta_{\max }^{*}$ are the solutions to (14) for $a(t)=0$ and $a(t)=1$, respectively.

When a muscle is passive, there exists only one solution $F^{m t}$ for a known $l^{m t}$. For $l^{m t} \leq l_{a, m i n}^{m t}$, for example, curve $F_{1}^{t}(\eta)$ in Figure 2, the solution to (14) results in a muscle length less than $0.5 l_{0}^{m}$ and tendon length less than its slack length $l_{s}^{t}$ such that the resulting muscle-tendon force is zero. For $l^{m t} \geq l_{a, \text { max }}^{m t}$, for example, curve $F_{5}^{t}(\eta)$ in Figure 2, the solution to (14) results in a muscle length greater than $1.5 l_{0}^{m}$ for the constraints (7) and (8) to be satisfied. Hence, the resulting passive muscle-tendon force can be expressed as

$$
F^{m t}=\left\{\begin{array}{cl}
0 & , l^{m t} \leq l_{a, \text { min }}^{m t} \\
F_{0}^{m} \hat{F}^{t}\left(\eta^{*}\right) & , l^{m t} \geq l_{a, \max }^{m t}
\end{array},\right.
$$

where $\eta^{*}$ is the solution to (14) for $a(t)=0$.

\section{Musculoskeletal Static Workspace Analysis}

The active muscle force range and passive muscle force provides the required actuation characteristics for the state dependent force generator as described in Section II-B. The muscle-tendon lengths are analogous to the lengths of the cables, and can be determined from the system kinematics. Using the muscle properties for a particular muscle, the minimum and maximum active lengths can be determined from (16) and (19), respectively. A cable is considered active if its length is between $l_{a, \text { min }}^{m t}<l^{m t}<l_{a, \text { max }}^{m t}$, otherwise it is regarded as a passive element.

The columns in $J^{T}$ for the active muscles form the columns of the transpose of the active Jacobian matrix $J_{a}^{T}$ in (4). The minimum and maximum active muscle forces from (20) for the set of active muscles form the vectors $\underline{\mathbf{f}}_{a}$ and $\overline{\mathbf{f}}_{a}$ within the workspace framework (5), respectively. Similarly, the columns in $J^{T}$ for the passive muscles and the passive muscle force from (21) form the columns of $J_{p}^{T}$ and elements of $\mathbf{f}_{p}$, respectively, within the static workspace defined in (5).

\section{SHOUldER MODEL}

To demonstrate the determination of muscle force range and musculoskeletal workspace analysis presented in Section
III, the static workspace is generated for a human shoulder. As shown in Figure 3(a), the human shoulder consists of the humerus bone (end-effector) that is connected to the scapularclavicle bone (base) through the glenohumeral joint. Accurate kinematics of the human shoulder were obtained from the well accepted OpenSim shoulder model developed by Holzbaur et al. [17], as shown in Figure 3(b). OpenSim is a widely accepted simulation platform in the biomechanics community used in performing analysis on musculoskeletal systems [18], for example, to study the muscle lengths and forces for a particular trajectory of motion.

The glenohumeral joint possesses three degrees-of-freedom and the pose of the system can be represented by $\mathbf{q}=$ $\left[\begin{array}{ccc}\alpha & \beta & \gamma\end{array}\right]^{T}$, where $\alpha, \beta$, and $\gamma$ are the $x y z$-Euler angles of the glenohumeral joint. Figure 3(a) shows the physiological interpretations for the pure rotations in $\alpha, \beta$ and $\gamma$.

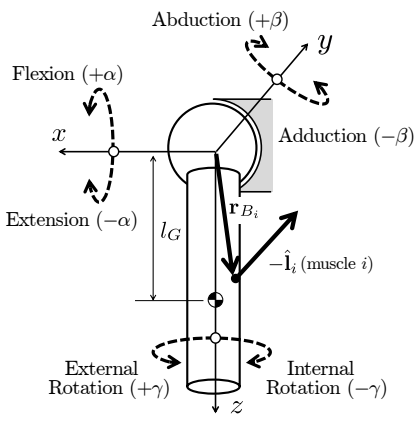

(a) Shoulder model kinematics

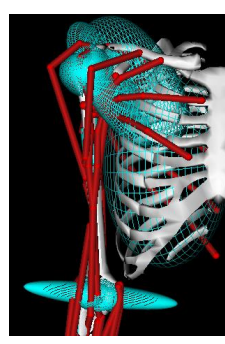

(b) OpenSim model
Fig. 3. Shoulder model consisting of the humerus bone and the glenohumeral joint.

The mass, inertia and location of the centre of gravity for the average human humerus were obtained from [19]. The muscles for the shoulder model consists of $m=15$ muscle sections that are identified as the main contributors to shoulder motion [17]: deltoid (anterior, middle, posterior), supraspinatus, infraspinatus, subscapularis, teres minor, teres major, pectoralis major (clavicular, sternal, ribs), latissimus dorsi (thoracic, lumbar, iliac) and coracobrachialis. The muscle properties $F_{0}^{m}, l_{0}^{m}, l_{s}^{t}$ and $\alpha_{0}$ for each muscle sub-region were obtained from [17].

For the shoulder system, the transpose of the Jacobian matrix can be expressed as

$$
J^{T}=\left[\begin{array}{llll}
\mathbf{r}_{B_{1}} \times \hat{\mathbf{l}}_{1} & \mathbf{r}_{B_{2}} \times \hat{\mathbf{l}}_{2} & \ldots & \mathbf{r}_{B_{15}} \times \hat{\mathbf{l}}_{15}
\end{array}\right] .
$$

As shown in Figure 3(a), the vectors $\mathbf{r}_{B_{i}}$ and $\hat{\mathbf{l}}_{i}$ represent the insertion location to the humerus and the direction vector of the insertion, respectively, for muscle $i$.

The kinematics of the human shoulder were obtained through the Holzbaur OpenSim model [17]. For a specified pose, the muscle-tendon length $l_{i}^{m t}$, insertion location $\mathbf{r}_{B_{i}}$ and direction vector of insertion $\hat{\mathbf{l}}_{i}$ of muscle $i$ could be obtained using the OpenSim API library. As a result, the generated workspace takes into consideration the complexities of human shoulder motion, such as kinematic constraints due to the glenohumeral joint, the wrapping of muscles, and the realistic movement of the scapula and clavicle bones. 
The musculoskeletal workspace was generated through the approach presented in Sections II-B and III-C. The search region $\mathcal{Q}$ was defined to be $\alpha \in\left[-180^{\circ}, 180^{\circ}\right], \beta \in\left[0,180^{\circ}\right]$ and $\gamma \in\left[-90^{\circ}, 90^{\circ}\right]$. The search region was uniformly discretised into a grid with a grid-size of $\Delta \alpha=\Delta \beta=\Delta \gamma=1^{\circ}$. To investigate the impact of the physiological muscle on the resulting workspace, the static workspace of an analogous traditional cable-driven manipulator for the same search region was generated through the approach presented in Section II-A. The insertion location $\mathbf{r}_{B_{i}}$ and muscle insertion direction $\hat{\mathbf{l}}_{i}$ for the muscles were obtained from the OpenSim API. The maximum force $f_{i, \max }$ for the ideal force generator cable $i$ was assumed to be the peak isometric force $F_{0}^{m}$ for muscle $i$.

\section{Musculos Keletal Static Workspace Results}

\section{A. Muscle Force Range}

To demonstrate the solution of muscle forces for different poses in $\mathcal{Q}$, the muscle forces for the deltoid (anterior) shoulder muscle is determined for flexion/extension motion. The muscle parameters for the deltoid (anterior) muscle are $F_{0}^{m}=1142.6 \mathrm{~N}, l_{0}^{m}=0.0976 \mathrm{~m}, l_{s}^{t}=0.093 \mathrm{~m}$ and $\alpha_{0}=0.384$. Flexion/extension motion refers to the poses $-180^{\circ} \leq \alpha \leq 180^{\circ}$ for $\beta=\gamma=0^{\circ}$. As the range of muscle forces varies depending on its muscle-tendon length, the muscle forces must be solved at each pose q. Figure 4 shows the resulting muscle-tendon forces for the deltoid (anterior) muscle during flexion/extension motion.

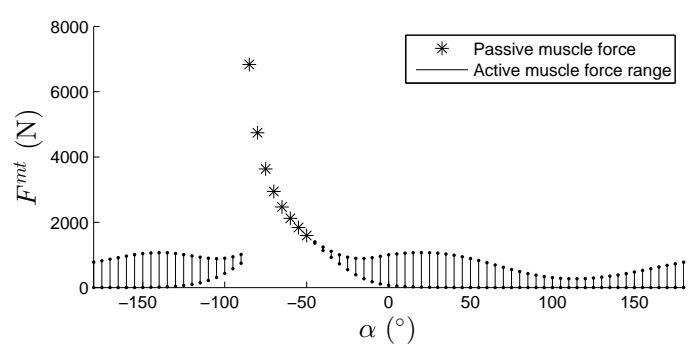

Fig. 4. Forces of deltoid (anterior) muscle during flexion/extension motion

Several features of the deltoid (anterior) muscle forces can be observed from Figure 4. Firstly, the muscle produces a varying range of forces at different flexion/extension angles. This corresponds with the natural behaviour of the state dependent force generator. Secondly, the deltoid is passive between $-85^{\circ} \leq \alpha \leq-50^{\circ}$ as $l^{m t} \geq l_{a, \max }^{m t}$, and the passive force is largest in magnitude at $\alpha=-85^{\circ}$. Finally, the deltoid is able to produce the largest range of active force at poses $\alpha=-140^{\circ}$ and $\alpha=15^{\circ}$, while at $\alpha=115^{\circ}, \alpha=-90^{\circ}$ and $-50^{\circ}<\alpha<-30^{\circ}$ the muscle is only able to produce a very limited range of forces.

\section{B. Musculoskeletal Static Workspace Analysis}

To determine the musculoskeletal static workspace, the muscle forces for the entire set of shoulder muscles were determined using the OpenSim API as for the deltoid (anterior) muscle previously presented. The musculoskeletal workspace is compared with the workspace for an analogous cable-driven
TABLE I

RANGE OF MOTION COMPARISON

\begin{tabular}{c|c|c|c}
\hline Motion & $\mathbf{S W}$ & $\mathbf{S W}^{\mathbf{M}}$ & Human Benchmark \\
\hline Flexion & $180^{\circ}$ & $158^{\circ}$ & $167^{\circ} \pm 9^{\circ}$ \\
Extension & $114^{\circ}$ & $29^{\circ}$ & $60^{\circ} \pm 7^{\circ}$ \\
Abduction & $180^{\circ}$ & $180^{\circ}$ & $179^{\circ} \pm 9^{\circ}$ \\
Horiz. Flexion & $180^{\circ}$ & $180^{\circ}$ & $138^{\circ} \pm 2^{\circ}$ \\
Horiz. Extension & $180^{\circ}$ & $90^{\circ}$ & $45^{\circ}$ \\
Horiz. Abduction & $180^{\circ}$ & $51^{\circ}$ & $45^{\circ}$ \\
\hline
\end{tabular}

system with ideal force generators. The $\alpha-\beta$ cross-sections of the workspace at $\gamma=0^{\circ}$ for both musculoskeletal and cable-driven shoulder systems are shown in Figure 5. The resolution of the workspace cross-sections have been displayed at $\Delta \alpha=\Delta \beta=5^{\circ}$ for visual clarity.

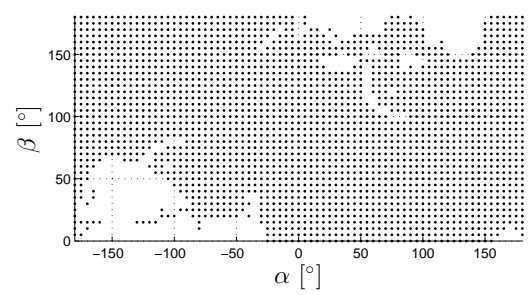

(a) Musculoskeletal workspace $S W^{M}$ at $\gamma=0^{\circ}$

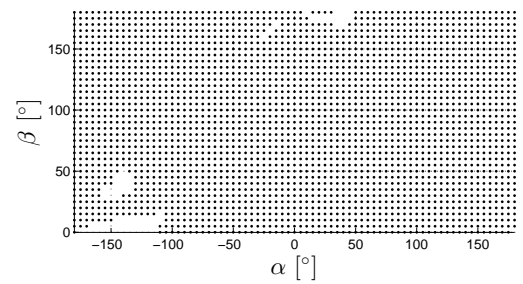

(b) Cable workspace $S W$ at $\gamma=0^{\circ}$

Fig. 5. $\alpha-\beta$ cross-sections of the static workspace for the shoulder system

From the $\alpha-\beta$ cross-sections, it can be observed that the workspace for the cable-driven system $S W$ with ideal force generators is significantly larger than that of the musculoskeletal system $S W^{M}$ with the Hill-type muscle model. The accuracy of the proposed musculoskeletal workspace is quantified by comparing the range of motion $(R O M)$ to that obtained from ROM benchmarks obtained from the literature [12], [13], [20], as shown in Table I.

The ROM limits for $S W^{M}$ and $S W$ were determined from the respective workspace boundaries. For example, the extension and flexion ROM limits are the minimum and maximum $\alpha$ values, respectively, of the workspace for zero rotation $\gamma=0$ and abduction $\beta=0$. From Figure 5(a), the ROM limits obtained from $S W^{M}$ for extension and flexion are $29^{\circ}$ and $158^{\circ}$, respectively. In comparison, the ROM obtained from the cable-driven system, as shown in Figure 5(b), for extension and flexion are $114^{\circ}$ and $180^{\circ}$, respectively. It should be noted that the ROM for internal and external rotations were not included in the ROM comparisons as it exceeded the range allowed by the Holzbaur model.

From Table I, it can be observed that the ROM from the musculoskeletal workspace $S W^{M}$ is more realistic compared 
TABLE II

WORKSPACE RANGE OF MOTION ERRORS COMPARED WITH BENCHMARK

\begin{tabular}{c|c|c}
\hline Property & $S W^{M}$ & $S W$ \\
\hline Mean error & $22.5^{\circ}$ & $63.2^{\circ}$ \\
Standard deviation of error & $19.4^{\circ}$ & $58.7^{\circ}$ \\
Range of error & $1^{\circ}-45^{\circ}$ & $1^{\circ}-135^{\circ}$ \\
\hline
\end{tabular}

to the benchmark human data than that from $S W$. Table II shows the mean and standard deviation of the error in the ROM of $S W$ and $S W^{M}$ compared with human benchmark data. It can be observed that the mean, standard deviation and range of error is significantly lower for $S W^{M}$ compared with $S W$.

\section{DISCUSSION}

Workspace analysis of biological systems can be regarded as an alternative and novel approach in studying the operational region of musculoskeletal systems. Compared with traditional experimental approaches in biomechanical studies, the proposed approach allows the complete region in which the system is considered as operational to be computationally generated. Obtaining the complete workspace region may be difficult to obtain experimentally, as it requires the human subject to perform the motion for all possible trajectories.

As a result, the proposed approach is beneficial in a range of biomechanics and biomechatronic applications. Increased understanding in the contribution of muscles to shoulder motion can be gained from the workspace analysis. For example, it can be observed from Figure 4 that the deltoid (anterior) muscle becomes passive for an extension of $\alpha=-50^{\circ}$, where the magnitude of passive force produced by the muscle becomes large. As a result, the range of force that can be produced by the muscle becomes very small in the region $-50^{\circ} \leq \alpha \leq$ $-30^{\circ}$. Hence, it predicted that the extension ROM limit of the shoulder is due to the limited force range of the deltoid (anterior) muscle. This is consistent with knowledge that the deltoid (anterior) muscle is known to be a major contributor to shoulder extension.

Furthermore, the computational approach allows the impact of changes in the system muscles on the workspace to be predicted. Changes in the system refer to the relocation of muscle attachments or variation in the muscle properties. For example, the effect of an injury, such as rotator cuff tear, can be studied by comparing the resulting workspace with that of a healthy subject. In tendon-transfer surgery, workspace analysis allows the effectiveness of the proposed muscle attachment relocations to be quantitatively evaluated.

\section{CONCLUSiOn AND Future Work}

The static workspace analysis for the human shoulder was performed by considering physiological muscles as state dependent force generators, where its ability to activate and produce force is dependent on the system pose. The condition for active and passive muscles, and its resulting muscle-tendon force, was expressed with respect to the system kinematics.
By comparing the computed shoulder workspace to the range of motion reported by human benchmarks, it was shown that the proposed approach is able to computationally produce a realistic workspace of the human shoulder. Future work will focus on the verification of the proposed approach on various musculoskeletal systems, such as the upper limb and the human neck.

\section{REFERENCES}

[1] S. K. Mustafa, G. Yang, S. H. Yeo, W. Lin, and I.-M. Chen, "Selfcalibration of a biologically inspired 7 DOF cable-driven robotic arm," IEEE/ASME Trans. Mechatron., vol. 13, no. 1, pp. 66-75, 2008.

[2] D. Lau, D. Oetomo, and S. K. Halgamuge, "Generalized modeling of multilink cable-driven manipulators with arbitrary routing using the cable-routing matrix," IEEE Trans. Robot., vol. 29, no. 5, pp. 1102$1113,2013$.

[3] T. Itto and K. Kogiso, "Hybrid modeling of mckibben pneumatic artificial muscle systems," in Proc. IEEE Int. Conf. on Industrial Technology, 2011, pp. 65-70.

[4] F. E. Zajac, "Muscle and tendon: Properties, models, scaling, and application to biomechanics and motor control," Crit. Rev. in Biomed. Eng., vol. 17, no. 4, pp. 359-411, 1989.

[5] Y. Guan, H. Zhang, X. Zhang, and Z. Guan, "Workspace generation of multi-fingered manipulation," Adv. Robot., vol. 25, no. 18, pp. 2293 2317, 2011.

[6] K. Gupta, "On the nature of robot workspace," Int. J. Robot. Res., vol. 5, no. 2, pp. 112-121, 1986.

[7] J. Yang, K. Abdel-Malek, and K. Nebel, "Reach envelope of a 9-degreeof-freedom model of the upper extremity," Int. J. Robot. Autom., vol. 20, no. 5, pp. 240-259, 2005

[8] J. Pusey, A. Fattah, S. Agrawal, and E. Messina, "Design and workspace analysis of a 6-6 cable-suspended parallel robot," Mech. Mach. Theory, vol. 39 , no. 7, pp. 761-778, 2004.

[9] D. Lau, D. Oetomo, and S. K. Halgamuge, "Wrench-closure workspace generation for cable driven parallel manipulators using a hybrid analytical-numerical approach," J. Mech. Des., vol. 133, no. 7, pp. $071004 / 1-7,2011$.

[10] M. Gouttefarde, D. Daney, and J.-P. Merlet, "Interval-analysis-based determination of the wrench-feasible workspace of parallel cable-driven robots," IEEE Trans. Robot., vol. 27, no. 1, pp. 1-13, 2011.

[11] L. Herda, R. Urtasun, P. Fua, and A. Hanson, "Automatic determination of shoulder joint limits using quarternion field boundaries," Int. J. Robot. Res., vol. 22, no. 6, pp. 419-436, 2003.

[12] D. C. Boone and S. P. Azen, "Normal range of motion of joints in male subjects," J. Bone Joint Surg., vol. 61, no. 5, pp. 756-759, 1979.

[13] G. Johnson, N. Fyfe, and M. Heward, "Ranges of movement at the shoulder complex using an electromagnetic movement sensor," Ann. Rheum. Dis., vol. 50, no. 11, pp. 824-827, 1991.

[14] C. F. Martin and L. Schovanec, "The control and mechanics of human movement systems," Progress in Systems and Control Theory, vol. 25, pp. 173-202, 1999.

[15] M. Vilimek, "Musculotendon forces derived by different muscle models," Acta of Bioeng. and Biomech., vol. 9, no. 2, pp. 41-47, 2007.

[16] S. H. Scott and D. A. Winter, "A comparison of three muscle pennation assumptions and their effects on isometric and isotonic force," $J$ Biomech., vol. 24, no. 2, pp. 163-167, 1991.

[17] K. R. S. Holzbaur, W. M. Murray, and S. L. Delp, "A model of the upper extremity for simulating musculoskeletal surgery and analyzing neuromuscular control," Ann. Biomed. Eng., vol. 33, no. 6, pp. 829840,2005

[18] S. L. Delp, F. C. Anderson, A. S. Arnold, P. Loan, A. Habib, C. T. John, E. Guendelman, and D. G. Thelen, "OpenSim: Open-source software to create and analyze dynamic simulations of movement," IEEE Trans. Biomed. Eng., vol. 54, no. 11, pp. 1940-1950, 2007.

[19] J. Yu, D. C. Ackland, and M. G. Pandy, "Shoulder muscle function depends on elbow joint position: An illustration of dynamic coupling in the upper limb," J. Biomech., vol. 44, no. 10, pp. 1859-1868, 2011.

[20] C. J. Barnes, S. V. Steyn, and R. A. Fischer, "The effects of age, sex and shoulder dominance on range of motion of the shoulder," J. Shoulder Elbow Surg., vol. 10, no. 3, pp. 242-246, 2001 